\title{
QUADRATIC PERTURBATIONS OF A QUADRATIC REVERSIBLE LOTKA-VOLTERRA SYSTEM
}

\author{
CHENGZHI LI ${ }^{1}$ AND JAUME LLIBRE ${ }^{2}$
}

\begin{abstract}
We prove that perturbing the two periodic annuli of the quadratic polynomial reversible Lotka-Volterra differential system $\dot{x}=$ $-y+x^{2}-y^{2}, \dot{y}=x(1+2 y)$, inside the class of all quadratic polynomial differential systems we can obtain the following configurations of limit cycles $(0,0),(1,0),(2,0),(1,1)$ and $(1,2)$.
\end{abstract}

\section{INTRODUCTION}

In this paper we consider the quadratic polynomial reversible LotkaVolterra differential system (in the classification of quadratic centers given by Żołądek [9] and Iliev [3]):

$$
\begin{aligned}
& \dot{x}=-y+x^{2}-y^{2}, \\
& \dot{y}=x(1+2 y) .
\end{aligned}
$$

On the finite plane this system has two singularities at $O(0,0)$ and $A(0,-1)$, both are centers. Between them there is the invariant straight line $\{(x, y)$ : $1+2 y=0\}$. The system has the integrating factor $\mu=1 /(1+2 y)^{2}$ and the rational first integral

$$
H(x, y)=\frac{x^{2}+(y+1)^{2}}{2(2 y+1)}=h, \quad \frac{1}{2}<h<\infty .
$$

System (1) is one of the four isochronous quadratic centers classified by Loud [5] in 1964.

1991 Mathematics Subject Classification. Primary 34C07, 34C08, 37G15.

Key words and phrases. quadratic vector field, limit cycles, isochronous center, averaging theory.

The first author is partially supported by NSFC-10671005 and NFSC-10831003. The second author is partially supported by MCYT grant MTM2005-06098-C02-01 and by a CIRIT grant number 2005SGR 00550. 
We shall perturb system (1) inside the class of all quadratic polynomial differential systems. Thus we consider systems of the form

$$
\begin{aligned}
& \dot{x}=-y+x^{2}-y^{2}+\varepsilon\left(a_{0}+a_{1} x+a_{2} y+a_{3} x^{2}+a_{4} x y+a_{5} y^{2}\right), \\
& \dot{y}=x(1+2 y)+\varepsilon\left(b_{0}+b_{1} x+b_{2} y+b_{3} x^{2}+b_{4} x y+b_{5} y^{2}\right),
\end{aligned}
$$

where $\varepsilon$ is small. We want to study how many limit cycles bifurcate from the periodic orbits of the period annuli of both centers for $\varepsilon$ sufficiently small.

Chicone and Jacobs in 1991 proved that up to first order it bifurcates at most one family of limit cycles from the period annulus surrounding the origin of system $(1)$, see for more details the isochronous $(-1 / 2,1 / 2)$ of $[2]$ (here we use the notation of the paper [2]). Later on, in 1998, Iliev [3] showed using abelian integrals of second order that at most two families of limit cycles bifurcate from that period annulus. Iliev did not get explicitly the function whose zeros provide the limit cycles in function of the coefficients of the perturbed system (3). Here we compute by first time this function using averaging theory of second order, and additionally we provide a new and different proof of the Chicone-Jacobs and Iliev results. See Section 2 for a short summary of the averaging theory applied to the computation of limit cycles. So our result is the following.

Theorem 1. The simple positive zeros of the function

$$
F(R)=\varepsilon A(R)+\varepsilon^{2} B(R)
$$

provide the number of limit cycles of system (3) for $\varepsilon$ sufficiently small, which can bifurcate from the period annulus surrounding the origin of system (1). Here

$$
A(R)=\left(b_{2}-\frac{b_{3}+b_{5}}{2}-2 b_{0}\right) R^{3}+\left(\frac{a_{1}+b_{2}}{2}-2 b_{0}\right) R .
$$

(a) If $A(R) \not \equiv 0$, then $F(R)$ has at most one positive zero. The limit cycle associated to this zero is the limit cycle obtained with the averaging theory of first order.

(b) If

$$
b_{2}=\frac{b_{3}+b_{5}}{2}+2 b_{0} \quad \text { and } \quad a_{1}=-\frac{b_{3}+b_{5}}{2}+2 b_{0}
$$

then

$$
B(R)=R\left(\alpha R^{2}+\beta\right)+\gamma \frac{\ln \left(R^{2}+1\right)}{R},
$$


where

$$
\begin{aligned}
& \alpha=\left(b_{4}-2 a_{3}+2 b_{1}-8 a_{0}-2 a_{5}+4 a_{2}\right) b_{3}+\left(4 b_{0}-b_{5}\right)\left(2 b_{1}-b_{4}\right), \\
& \beta=\left(2 a_{4}-4 b_{3}\right) a_{0}+\left(-4 a_{3}+8 b_{1}-2 b_{4}\right) b_{0}-\gamma, \\
& \gamma=\left(a_{4}+2 b_{3}\right)\left(b_{1}-a_{3}-a_{5}+a_{2}\right) .
\end{aligned}
$$

Now the positive zeros of $F(R)$ are those of $B(R)$. So $F(R)$ has at most two positive zeros. The limit cycles associated to these zeros are the limit cycle obtained with the averaging theory of second order.

Theorem 1 is proved in Sections 3 and 4 .

Since system (1) has two centers, one at the origin and the other at the point $(0,-1)$ when we perturb it as in (3) they can bifurcate limit cycles from the period annuli of both centers. We say that system (3) for $\varepsilon$ sufficiently small has the configuration $(i, j)$ of limit cycles if from one period annulus bifurcate $i$ limit cycles and from the other $j$. We shall prove in Section 5 the following result.

Theorem 2. System (3) for $\varepsilon$ sufficiently small can exhibit the following configurations of limit cycles $(0,0),(1,0),(2,0),(1,1)$ and $(1,2)$.

\section{PeRIOdic SOlutions FOR PERIODIC DIFFERENTIAL EQUATIONS VIA \\ AVERAGING THEORY}

In this section we summarize the main results on the averaging theory that we will apply to our quadratic polynomial differential systems (1).

The next theorem provides a first order approximation for the periodic solutions of a periodic differential system, for a proof see Theorem 2.6.1 of Sanders Verhulst [6] and Theorems 11.5 and 11.6 of Verhulst [7]. The original theorems are given for a system of differential equations, but since we will use them only for one differential equation we state them in this case.

Theorem 3. Consider the following two initial value problems

$$
\dot{x}=\varepsilon f(t, x)+\varepsilon^{2} h(t, x, \varepsilon), \quad x(0)=x_{0},
$$

and

$$
\dot{y}=\varepsilon f^{0}(y), \quad y(0)=x_{0},
$$

where $x, y, x_{0} \in D, D$ is an open subset of $\mathbb{R}, t \in[0, \infty), \varepsilon \in\left(0, \varepsilon_{0}\right], f$ and $h$ are periodic of period $T$ in $t$, and

$$
f^{0}(y)=\frac{1}{T} \int_{0}^{T} f(t, y) d t
$$

Suppose that 
(i) $f, \partial f / \partial x, \partial^{2} f / \partial x^{2}$ and $\partial h / \partial x$ are defined, continuous and bounded by a constant independent on $\varepsilon$ in $[0, \infty) \times D$ and $\varepsilon \in\left(0, \varepsilon_{0}\right]$;

(ii) $T$ is independent on $\varepsilon$; and

(iii) $y(t)$ belongs to $D$ on the time-scale $1 / \varepsilon$.

Then the following statements hold.

(a) On the time-scale $1 / \varepsilon$ we have that

$$
x(t)-y(t)=O(\varepsilon), \quad \text { as } \quad \varepsilon \rightarrow 0 .
$$

(b) If $p$ is an equilibrium point of the averaging system (6) such that

$$
\left(\partial f^{0} / \partial y\right)(p) \neq 0
$$

then there exists a $T$-periodic solution $\phi(t, \varepsilon)$ of equation (5) which is close to $p$ such that $\phi(t, \varepsilon) \rightarrow p$ as $\varepsilon \rightarrow 0$.

(c) If (8) is negative, then the corresponding periodic solution $\phi(t, \varepsilon)$ in the space $(t, x)$ is asymptotically stable for $\varepsilon$ sufficient small. If (8) is positive, then it is unstable.

The next theorem provides a second order approximation for the solutions of a periodic differential system, for a proof see Theorem 3.5.1 of Sanders and Verhulst [6], and Corollary 6 of [4].

Theorem 4. Consider the following two initial value problems

$$
\dot{x}=\varepsilon f(t, x)+\varepsilon^{2} g(t, x)+\varepsilon^{3} h(t, x, \varepsilon), \quad x(0)=x_{0},
$$

and

$$
\dot{y}=\varepsilon f^{0}(y)+\varepsilon^{2} f^{10}(y)+\varepsilon^{2} g^{0}(y), \quad y(0)=x_{0},
$$

with $f, g:[0, \infty) \times D \rightarrow G:[0, \infty) \times D \times\left(0, \varepsilon_{0}\right] \rightarrow \mathbb{R}, D$ an open subset of $\mathbb{R}, f, g$ and $h$ periodic of period $T$ in $t$, and

$$
f^{1}(t, x)=\frac{\partial f}{\partial x} y^{1}(t, x)-\frac{\partial y^{1}}{\partial x} f^{0}(x)
$$

where

$$
y^{1}(t, x)=\int_{0}^{t}\left(f(s, x)-f^{0}(x)\right) d s+z(x),
$$

with $z(x)$ a $C^{1}$ function such that the averaging of $y^{1}$ is zero. Besides, $f^{0}, f^{10}$ and $g^{0}$ denote the averaging functions of $f, f^{1}$ and $g$, respectively, defined as in (6). Suppose that

(i) $\partial f / \partial x$ is Lipschitz in $x$ and all these functions are continuous on their domain of definition;

(ii) $|h(t, x, \varepsilon)|$ is bounded by a constant uniformly in $[0, L / \varepsilon) \times D \times\left(0, \varepsilon_{0}\right]$;

(iii) $T$ is independent on $\varepsilon$; and

(iv) $y(t)$ belongs to $D$ on the time-scale $1 / \varepsilon$. 
Then

(a) On the time-scale $1 / \varepsilon$ we have that

$$
x(t)=y(t)+\varepsilon y^{1}(t, y(t))+O\left(\varepsilon^{2}\right), \quad \text { as } \varepsilon \rightarrow 0 .
$$

If, in addition, $f^{0}(y) \equiv 0$, then the following statements hold.

(b) If $p$ is an equilibrium of the averaging system (10) such that

$$
\frac{\partial}{\partial y}\left(f^{10}(y)+g^{0}(y)\right)(p) \neq 0
$$

then there exists a $T$-periodic solution $\phi(t, \varepsilon)$ of equation (9) which is close to $p$ such that $\phi(t, \varepsilon) \rightarrow p$ as $\varepsilon \rightarrow 0$.

(c) If (13) is negative, then the corresponding periodic solution $\phi(t, \varepsilon)$ in the space $(t, x)$ is asymptotically stable for $\varepsilon$ sufficient small. If (13) is positive, then it is unstable.

We consider a planar integrable system of the form

$$
\dot{x}=P(x, y), \quad \dot{y}=Q(x, y),
$$

with an integrating factor $\mu(x, y) \neq 0$, a first integral $H$, and a continuous family of ovals

$$
\left\{\gamma_{h}\right\} \subset\left\{(x, y): H(x, y)=h, h_{1}<h<h_{2}\right\} .
$$

We perturb this system as follows

$$
\begin{aligned}
& \dot{x}=P(x, y)+\varepsilon p(x, y), \\
& \dot{y}=Q(x, y)+\varepsilon q(x, y) .
\end{aligned}
$$

In order to study the number of limit cycles of system (15) for sufficiently small $\varepsilon$ by using the above averaging theorems, we need to transform system (15) to the canonical forms (5) or (9) for applying the averaging theory. The following result of [1] provides a way for a such transformation.

Theorem 5. Assume that $x Q(x, y)-y P(x, y) \neq 0$ for all $(x, y)$ in the period annulus formed by the ovals $\left\{\gamma_{h}\right\}$. Let $\rho:\left(\sqrt{h_{1}}, \sqrt{h_{2}}\right) \times[0,2 \pi) \rightarrow[0, \infty)$ be a continuous function such that

$$
H(\rho(R, \varphi) \cos \varphi, \rho(R, \varphi) \sin \varphi)=R^{2},
$$

for all $R \in\left(\sqrt{h_{1}}, \sqrt{h_{2}}\right)$ and all $\varphi \in[0,2 \pi)$. Then the differential equation which describes the dependence between the square root of energy, $R=\sqrt{h}$, and the angle $\varphi$ for system (15) is

$$
\frac{d R}{d \varphi}=\varepsilon \frac{\mu\left(x^{2}+y^{2}\right)(Q p-P q)}{2 R(Q x-P y)}\left(1-\varepsilon \frac{q x-p y}{Q x-P y}\right)+O\left(\varepsilon^{3}\right),
$$

where $x=\rho(R, \varphi) \cos \varphi$ and $y=\rho(R, \varphi) \sin \varphi$. 


\section{Bifurcation Function}

Let

$$
\rho=\rho(\varphi, R)=R\left(R \sin \varphi+\sqrt{R^{2} \sin ^{2} \varphi+1}\right),
$$

then from (2) we have

$$
H(\rho(\varphi, R) \cos \varphi, \rho(\varphi, R) \sin \varphi)=\frac{R^{2}+1}{2}, \quad 0<R<\infty .
$$

Comparing the right hand side of (19) with (16), we use $\left(R^{2}+1\right) / 2$ instead of $R^{2}$, this makes the only difference of removing the factor 2 from the denominator of the right hand side of formula (17). We define

(20) $F(x, y)=\frac{F_{1}(x, y)}{R(y+1)(2 y+1)^{2}}, \quad G(x, y)=-F(x, y) \frac{G_{1}(x, y)}{\left(x^{2}+y^{2}\right)(y+1)}$,

where

$$
\begin{aligned}
F_{1}= & -b_{3} x^{4}+\left(2 a_{3}-b_{4}\right) x^{3} y+\left(2 a_{4}+b_{3}-b_{5}\right) x^{2} y^{2}+\left(2 a_{5}+b_{4}\right) x y^{3} \\
& +b_{5} y^{4}+\left(a_{3}-b_{1}\right) x^{3}+\left(2 a_{1}+a_{4}-b_{2}+b_{3}\right) x^{2} y \\
& +\left(2 a_{2}+a_{5}+b_{1}+b_{4}\right) x y^{2}+\left(b_{2}+b_{5}\right) y^{3}+\left(a_{1}-b_{0}\right) x^{2} \\
& +\left(a_{2}+b_{1}+2 a_{0}\right) x y+\left(b_{2}+b_{0}\right) y^{2}+a_{0} x+b_{0} y \\
G_{1}= & b_{3} x^{3}+\left(b_{4}-a_{3}\right) x^{2} y+\left(b_{5}-a_{4}\right) x y^{2}-a_{5} y^{3}+b_{1} x^{2} \\
& +\left(b_{2}-a_{1}\right) x y-a_{2} y^{2}+b_{0} x-a_{0} y .
\end{aligned}
$$

Then by using Theorem 5 we transform system (3) into the form

$$
\frac{d R}{d \varphi}=f(\varphi, R) \varepsilon+g(\varphi, R) \varepsilon^{2}+O\left(\varepsilon^{3}\right),
$$

where

$$
\begin{aligned}
& f=F(\rho(\varphi, R) \cos \varphi, \rho(\varphi, R) \sin \varphi), \\
& g=G(\rho(\varphi, R) \cos \varphi, \rho(\varphi, R) \sin \varphi) .
\end{aligned}
$$

In order to use Theorem 3 we need to computer the first averaging function $f^{0}(R)$ defined by

$$
f^{0}(R)=\frac{1}{2 \pi} \int_{0}^{2 \pi} f(\varphi, R) d \varphi .
$$

From (22), (20) and (18) we find that

$$
f(\varphi, R)=f_{1}(\varphi, R)+\frac{f_{2}(\varphi, R)}{\sqrt{R^{2} \sin ^{2} \varphi+1}},
$$


where

$$
\begin{aligned}
f_{1}=R & {\left[\left(a_{1}-b_{0}-b_{3} R^{2}\right) \cos ^{2} \varphi+\left(a_{2}+b_{1}-2 a_{0}+\left(2 b_{1}-b_{4}\right) R^{2}\right) \sin \varphi \cos \varphi\right.} \\
& \left.+\left(b_{2}-3 b_{0}+\left(2 b_{2}-b_{5}-4 b_{0}\right) R^{2}\right) \sin ^{2} \varphi\right], \\
f_{2}=[ & \left.\left(-2 b_{1}+b_{4}\right) R^{4}+\left(b_{4}-a_{2}+a_{5}-2 b_{1}+2 a_{0}\right) R^{2}+a 0\right] \cos \varphi \\
& +R^{2}\left[\left(2 b_{1}-b_{4}\right) R^{2}-a_{5}+b_{1}-b_{4}+a_{2}-2 a_{0}+a_{3}\right] \cos ^{3} \varphi \\
& +\left[b_{3} R^{4}+\left(-a_{1}+b_{3}+3 b_{0}+a_{4}-b_{2}\right) R^{2}+b_{0}\right] \sin \varphi \\
& -R^{2}\left[\left(2 b_{2}-4 b_{0}+b_{3}-b_{5}\right) R^{2}+a_{4}-a_{1}+b_{3}-2 b_{0}+b_{2}-b_{5}\right] \sin ^{3} \varphi .
\end{aligned}
$$

Hence, by direct computation we obtain

$$
\begin{aligned}
f^{0}(R) & =\frac{1}{2 \pi} \int_{0}^{2 \pi} f_{1}(\varphi, R) d \varphi \\
& =\left(b_{2}-\frac{b_{3}+b_{5}}{2}-2 b_{0}\right) R^{3}+\left(\frac{a_{1}+b_{2}}{2}-2 b_{0}\right) R
\end{aligned}
$$

From this result we find that $f^{0}(R)$ has at most one zero for $R>0$. Since system (1) is in the intersection of reversible and generalized Lotka-Volterra systems, the first order averaging could not give a maximal number of limit cycles of the perturbed system. So we will use Theorem 4 to compute the second order averaging. Hence, we take $f^{0}(R) \equiv 0$, that is

$$
b_{2}=\frac{b_{3}+b_{5}}{2}+2 b_{0}, \quad a_{1}=-\frac{b_{3}+b_{5}}{2}+2 b_{0} .
$$

Substituting (26) into (24), we obtain the new expressions of $f_{1}$ and $f_{2}$ as follows.

$$
\begin{aligned}
f_{1}= & R\left[\left(\frac{b_{3}+b_{5}}{2}-b_{0}+b_{3} R^{2}\right)\left(\sin ^{2} \varphi-\cos ^{2} \varphi\right)\right. \\
& \left.+\left(a_{2}+b_{1}-2 a_{0}+\left(2 b_{1}-b_{4}\right) R^{2}\right) \sin \varphi \cos \varphi\right], \\
f_{2}= & \alpha_{11} \cos \varphi+\alpha_{13} \cos ^{3} \varphi+\beta_{11} \sin \varphi+\beta_{13} \sin ^{3} \varphi
\end{aligned}
$$

where

$$
\begin{aligned}
& \alpha_{11}=a_{0}+\left(b_{4}+a_{5}+2 a_{0}-a_{2}-2 b_{1}\right) R^{2}+\left(b_{4}-2 b_{1}\right) R^{4}, \\
& \alpha_{13}=\left[a_{2}+a_{3}+b_{1}-b_{4}-a_{5}-2 a_{0}+\left(2 b_{1}-b_{4}\right) R^{2}\right] R^{2}, \\
& \beta_{11}=b_{0}+\left(b_{3}+a_{4}-b_{0}\right) R^{2}+b_{3} R^{4}, \\
& \beta_{13}=\left[2 b_{0}-a_{4}-2 b_{3}-2 b_{3} R^{2}\right] R^{2} .
\end{aligned}
$$


To compute the function $f^{1}(\varphi, R)$ given in (11), we first need to find $y^{1}(\varphi, R)$ given in (12). Direct computation gives

$$
\begin{aligned}
\int \frac{\cos \varphi}{\sqrt{R^{2} \sin ^{2} \varphi+1}} d \varphi= & \frac{1}{R} \ln \left(R \sin \varphi+\sqrt{R^{2} \sin ^{2} \varphi+1}\right) \\
\int \frac{\cos ^{3} \varphi}{\sqrt{R^{2} \sin ^{2} \varphi+1}} d \varphi= & -\frac{\sin \varphi \sqrt{R^{2} \sin ^{2} \varphi+1}}{2 R^{2}} \\
& +\frac{2 R^{2}+1}{2 R^{3}} \ln \left(R \sin \varphi+\sqrt{R^{2} \sin ^{2} \varphi+1},\right) \\
\int \frac{\sin \varphi}{\sqrt{R^{2} \sin ^{2} \varphi+1}} d \varphi= & -\frac{1}{R} \arctan \frac{R \cos \varphi}{\sqrt{R^{2} \sin ^{2} \varphi+1}}, \\
\int \frac{\sin ^{3} \varphi}{\sqrt{R^{2} \sin ^{2} \varphi+1}} d \varphi= & -\frac{\cos \varphi \sqrt{R^{2} \sin ^{2} \varphi+1}}{2 R^{2}}+\frac{1-R^{2}}{2 R^{3}} \arctan \frac{R \cos \varphi}{\sqrt{R^{2} \sin ^{2} \varphi+1}}
\end{aligned}
$$

From these expressions and using some symmetries we obtain

$$
\begin{aligned}
y^{1}(\varphi, R)= & \gamma_{1} \sin ^{2} \varphi+\gamma_{2} \sin \varphi \cos \varphi+\gamma_{3} \\
& -\frac{\left(\alpha_{13} \sin \varphi+\beta_{13} \cos \varphi\right) \sqrt{R^{2} \sin ^{2} \varphi+1}}{2 R^{2}} \\
& +\gamma_{4} \ln \left(R \sin \varphi+\sqrt{R^{2} \sin ^{2} \varphi+1}\right) \\
& +\gamma_{5} \arctan \frac{R \cos \varphi}{\sqrt{R^{2} \sin ^{2} \varphi+1}},
\end{aligned}
$$

where

$$
\begin{aligned}
\gamma_{1} & =\frac{1}{2}\left[a_{2}+b_{1}-2 a_{0}+\left(2 b_{1}-b_{4}\right) R^{2}\right] R, \\
\gamma_{2} & =-\frac{1}{2}\left(b_{3}+b_{5}-2 b_{0}+2 b_{3} R^{2}\right) R \\
\gamma_{3} & =-\frac{1}{4}\left[b_{1}+a_{2}-2 a_{0}+\left(2 b_{1}-b_{4}\right) R^{2}\right] R, \\
\gamma_{4} & =\frac{\alpha_{11}}{R}+\frac{\alpha_{13}\left(2 R^{2}+1\right)}{2 R^{3}} \\
\gamma_{5} & =-\frac{\beta_{11}}{R}+\frac{\beta_{13}\left(1-R^{2}\right)}{2 R^{3}} .
\end{aligned}
$$

By using (11) and making averaging we have:

$$
f^{10}(R)=\frac{1}{2 \pi} \int_{0}^{2 \pi} y^{1}(\varphi, R) \frac{\partial f(\varphi, R)}{\partial R} d \varphi .
$$


Note that

$$
\frac{\partial f(\varphi, R)}{\partial R}=M_{1}(\varphi, R)+\frac{M_{2}(\varphi, R)}{\left(R^{2} \sin ^{2} \varphi+1\right)^{3 / 2}},
$$

where

$$
\begin{aligned}
M_{1}= & \left(\frac{b_{3}+b_{5}}{2}-b_{0}+3 b_{3} R^{2}\right)\left(\sin ^{2} \varphi-\cos ^{2} \varphi\right) \\
& +\left(a_{2}+b_{1}-2 a_{0}+3\left(2 b_{1}-b_{4}\right) R^{2}\right) \sin \varphi \cos \varphi, \\
M_{2}= & m_{21} \cos \varphi+m_{22} \cos ^{3} \varphi+m_{23} \cos ^{5} \varphi+m_{24} \sin \varphi+m_{25} \sin ^{3} \varphi+m_{26} \sin ^{5} \varphi,
\end{aligned}
$$

with

$$
\begin{aligned}
m_{21}= & {\left[\left(3 b_{4}-6 b 1\right) R^{4}-\left(a_{2}-a_{5}-2 a_{0}-5 b_{4}+10 b_{1}\right) R^{2}\right.} \\
& \left.+3 a_{0}-2 a_{2}+2 b_{4}+2 a_{5}-4 b_{1}\right] R, \\
m_{22}= & {\left[6\left(2 b_{1}-b_{4}\right) R^{4}+\left(2 a_{2}-4 a_{0}+11 b_{1}-6 b_{4}+a_{3}-2 a_{5}\right) R^{2}\right.} \\
& \left.-2 a_{5}-3 a_{0}+2 a_{2}+2 a_{3}-2 b_{4}+2 b_{1}\right] R, \\
m_{23}= & {\left[3\left(b_{4}-2 b_{1}\right) R^{2}-a_{3}+2 a_{0}-b_{1}+a_{5}+b_{4}-a_{2}\right] R^{3}, } \\
m_{24}= & 2\left(2 b_{3} R^{2}+b_{3}-b_{0}+a_{4}\right) R, \\
m_{25}= & {\left[3 b_{3} R^{4}+\left(a_{4}-b_{0}-7 b_{3}\right) R^{2}+3 b_{0}-4 b_{3}-2 a_{4}\right] R, } \\
m_{26}= & \left(-6 b_{3} R^{2}+2 b_{0}-a_{4}-2 b_{3}\right) R^{3} .
\end{aligned}
$$

Substituting (27), (28) and (30) into (29), and using the symmetry property, we have

$$
f^{10}(R)=W_{1}(R)+W_{2}(R)+W_{3}(R)
$$

where

$$
\begin{aligned}
& W_{1}(R)=\frac{1}{2 \pi} \int_{0}^{2 \pi}\left(\gamma_{1} \sin ^{2} \varphi+\gamma_{2} \sin \varphi \cos \varphi+\gamma_{3}\right) M_{1}(\varphi, R) d \varphi \\
& W_{2}(R)=-\frac{1}{4 \pi R^{2}} \int_{0}^{2 \pi} \frac{\left(\alpha_{13} \sin \varphi+\beta_{13} \cos \varphi\right) M_{2}(\varphi, R)}{R^{2} \sin ^{2} \varphi+1} d \varphi \\
& W_{3}(R)=\frac{1}{2 \pi}\left[\gamma_{4}\left(m_{24} A_{1}+m_{25} A_{3}+m_{26} A_{5}\right)+\gamma_{5}\left(m_{21} B_{1}+m_{22} B_{3}+m_{23} B_{5}\right)\right]
\end{aligned}
$$

with

$$
\begin{aligned}
& A_{j}(R)=\int_{0}^{2 \pi} \frac{\sin ^{j} \varphi \ln \left(R \sin \varphi+\sqrt{R^{2} \sin ^{2} \varphi+1}\right)}{\left(R^{2} \sin ^{2} \varphi+1\right)^{3 / 2}} d \varphi, \quad j=1,3,5, \\
& B_{j}(R)=\int_{0}^{2 \pi} \frac{\cos ^{j} \varphi}{\left(R^{2} \sin ^{2} \varphi+1\right)^{3 / 2}} \arctan \frac{R \cos \varphi}{\sqrt{R^{2} \sin ^{2} \varphi+1}} d \varphi, \quad j=1,3,5 .
\end{aligned}
$$

To compute $A_{j}(R)$ and $B_{j}(R)$, we need the following integrals, which were obtained by using the technique to make a derivative of an integral 
with resect to a parameter, and solve a differential equation satisfied by the integral.

$$
\begin{aligned}
I_{0}(R) & =\int_{0}^{2 \pi}\left[\sin \varphi\left(R^{2} \sin ^{2} \varphi+1\right)^{1 / 2} \ln \left(R \sin \varphi+\sqrt{R^{2} \sin ^{2} \varphi+1}\right)\right] d \varphi \\
& =\frac{\pi}{2 R}\left[\left(R^{2}+1\right) \ln \left(R^{2}+1\right)+R^{2}\right] \\
I_{1}(R) & =\int_{0}^{2 \pi} \frac{\left[\sin \varphi \ln \left(R \sin \varphi+\sqrt{R^{2} \sin ^{2} \varphi+1}\right)\right]}{\left(R^{2} \sin ^{2} \varphi+1\right)^{1 / 2}} d \varphi=\frac{\pi}{R} \ln \left(R^{2}+1\right), \\
I_{3}(R) & =\int_{0}^{2 \pi} \frac{\left[\sin \varphi \ln \left(R \sin \varphi+\sqrt{R^{2} \sin ^{2} \varphi+1}\right)\right]}{\left(R^{2} \sin ^{2} \varphi+1\right)^{3 / 2}} d \varphi=\frac{2 \pi\left(\sqrt{R^{2}+1}-1\right)}{R\left(R^{2}+1\right)} \\
J_{0}(R) & =\int_{0}^{2 \pi} \cos \varphi\left(R^{2} \sin ^{2} \varphi+1\right)^{1 / 2} \arctan \frac{R \cos \varphi}{\sqrt{R^{2} \sin { }^{2} \varphi+1}} d \varphi \\
& =\frac{\pi}{2 R}\left(\ln \left(R^{2}+1\right)+R^{2}\right), \\
J_{1}(R) & =\int_{0}^{2 \pi} \frac{\cos \varphi}{\left(R^{2} \sin ^{2} \varphi+1\right)^{1 / 2}} \arctan \frac{R \cos \varphi}{\sqrt{R^{2} \sin ^{2} \varphi+1}} d \varphi=\frac{\pi}{R} \ln \left(R^{2}+1\right), \\
J_{3}(R) & =\int_{0}^{2 \pi} \frac{\cos \varphi}{\left(R^{2} \sin ^{2} \varphi+1\right)^{3 / 2}} \arctan \frac{R \cos \varphi}{\sqrt{R^{2} \sin ^{2} \varphi+1}} d \varphi=\frac{2 \pi\left(\sqrt{R^{2}+1}-1\right)}{R \sqrt{R^{2}+1}} .
\end{aligned}
$$

An elementary computation gives

$$
\begin{aligned}
& A_{1}=I_{3}, \\
& A_{3}=\left(I_{1}-I_{3}\right) / R^{2}, \\
& A_{5}=\left(I_{0}-2 I_{1}+I_{3}\right) / R^{4}, \\
& B_{1}=J_{3}, \\
& B_{3}=\left(\left(R^{2}+1\right) J_{3}-J_{1}\right) / R^{2}, \\
& B_{5}=\left(\left(R^{2}+1\right)^{2} J_{3}-2\left(R^{2}+1\right) J_{1}+J_{0}\right) / R^{4} .
\end{aligned}
$$

Now we may give the results for $f^{10}(R)=W_{1}(R)+W_{2}(R)+W_{3}(R)$, but we do not write down it, because the expression is very long. Once we finish the calculation of $g^{0}(R)$, we will find that the expression for $f^{10}(R)+g^{0}(R)$ is very simple. The whole calculations can be done by using some algebraic manipulator as Maple or Mathematica. 
From $(21)$ and $(20)$ we can find the expression for $g(\varphi, R)$ which is given

$g(\varphi, R)=\frac{1}{R}\left(f_{1}(\varphi, R)+\frac{f_{2}(\varphi, R)}{\sqrt{R^{2} \sin ^{2} \varphi+1}}\right)\left(g_{1}(\varphi, R)+\frac{g_{2}(\varphi, R)}{\sqrt{R^{2} \sin ^{2} \varphi+1}}\right)$,

where $f_{1}$ and $f_{2}$ are the same as in (27), and $g_{1}$ and $g_{2}$ are as follows:

$$
\begin{aligned}
g_{1}= & {\left[-b_{1} \cos ^{2} \varphi+\left(2 b_{0}-b 3-b_{5}\right) \sin \varphi \cos \varphi+\left(a_{2}-2 a_{0}\right) \sin ^{2} \varphi\right] R, } \\
g_{2}= & R^{2}\left(2 b_{0}-2 b_{3}-a_{4}\right) \cos ^{3} \varphi+\left(\left(a_{4}-2 b_{0}+b_{3}\right) R^{2}-b_{0}\right) \cos \varphi \\
& +R^{2}\left(2 a_{0}-a_{2}+a_{5}+b_{4}-b_{1}-a_{3}\right) \sin ^{3} \varphi+\left(\left(a_{3}-b_{4}+b_{1}\right) R^{2}+a_{0}\right) \sin \varphi .
\end{aligned}
$$

Hence, we have

$$
g^{0}(R)=\frac{1}{2 \pi R}\left(\int_{0}^{2 \pi} f_{1}(\varphi, R) g_{1}(\varphi, R) d \varphi+\int_{0}^{2 \pi} \frac{f_{2}(\varphi, R) g_{2}(\varphi, R)}{R^{2} \sin ^{2} \varphi+1} d \varphi\right) .
$$

Finally, from (31) and 33) we obtain

$$
B(R)=f^{10}(R)+g^{0}(R)=R\left(\alpha R^{2}+\beta\right)+\gamma \frac{\ln \left(R^{2}+1\right)}{R},
$$

where

$$
\begin{aligned}
& \alpha=\left(b_{4}-2 a_{3}+2 b_{1}-8 a_{0}-2 a_{5}+4 a_{2}\right) b_{3}+\left(4 b_{0}-b_{5}\right)\left(2 b_{1}-b_{4}\right) \\
& \beta=\left(2 a_{4}-4 b_{3}\right) a_{0}+\left(-4 a_{3}+8 b_{1}-2 b_{4}\right) b_{0}-\gamma \\
& \gamma=\left(a_{4}+2 b_{3}\right)\left(b_{1}-a_{3}-a_{5}+a_{2}\right)
\end{aligned}
$$

\section{Discussion of the Bifurcation Function}

From (34) we have that the Jacobian

$$
\frac{D(\alpha, \beta, \gamma)}{D\left(a_{5}, b_{3}, b_{5}\right)}=4\left(b_{4}-2 b_{1}\right)\left(a_{4}+2 b_{3}\right) a_{0}
$$

Hence, if we choose $\left(b_{4}-2 b_{1}\right)\left(a_{4}+2 b_{3}\right) a_{0} \neq 0$, the coefficients in the bifurcation function $B(R)$ can be chosen freely. Since we may divide $B(R)$ by one of non-zero coefficients, the number of independent coefficients actually is two.

In fact, if $\gamma=0$, then $B(R)$ has at most one positive zero; if $\gamma \neq 0$, to study the number of its positive zeros, we may change $B(R)$ into the form

$$
B_{1}(R)=\gamma\left(R^{2}\left(a R^{2}+b\right)+\ln \left(R^{2}+1\right)\right), \quad 0 \leq R<+\infty .
$$

Since

$$
B_{1}^{\prime}(R)=\frac{\gamma}{R^{2}+1}\left(2 a R^{4}+(2 a+b) R^{2}+1+b\right)
$$


it has at most 2 positive zeros, implying $B(R)$ has at most two positive zeros.

On the other hand, if we choose $a=0.02$ and $b=-0.5$, then $B_{1}(R)$ (hence $B(R)$ ) has exact 2 positive zeros at $R_{1} \approx 1.869, R_{2} \approx 4.0287$.

\section{Limit cycles Surrounding Another Center}

To consider the bifurcation from another annulus surrounding the center at $(x, y)=(0,-1)$, if we keep the condition $(26)$ and make changing $(x, y) \mapsto$ $(x, u-1)$, followed by $(x, u) \mapsto(x,-y)$, then we obtain

$$
\begin{aligned}
& \dot{x}=-y+x^{2}-y^{2}+\varepsilon\left(\bar{a}_{0}+\bar{a}_{1} x+\bar{a}_{2} y+\bar{a}_{3} x^{2}+\bar{a}_{4} x y+\bar{a}_{5} y^{2}\right), \\
& \dot{y}=x(1+2 y)+\varepsilon\left(\bar{b}_{0}+\bar{b}_{1} x+\bar{b}_{2} y+\bar{b}_{3} x^{2}+\bar{b}_{4} x y+\bar{b}_{5} y^{2}\right),
\end{aligned}
$$

where

$$
\begin{array}{ll}
\bar{a}_{0}=a_{0}+a_{5}-a_{2}, & \bar{a}_{1}=2 b_{0}-\frac{b_{3}}{2}-\frac{b_{5}}{2}-a_{4}, \\
\bar{a}_{2}=\left(2 a_{5}-a_{2}\right), & \bar{a}_{3}=a_{3}, \\
\bar{a}_{4}=-a_{4}, & \bar{a}_{5}=a_{5}, \\
\bar{b}_{0}=b_{0}+\frac{b_{3}}{2}-\frac{b_{5}}{2}, & \bar{b}_{1}=b_{4}-b_{1}, \\
\bar{b}_{2}=2 b_{0}+\frac{b_{3}}{2}-\frac{3 b_{5}}{2}, & \bar{b}_{3}=-b_{3}, \\
\bar{b}_{4}=b_{4}, & \bar{b}_{5}=-b_{5} .
\end{array}
$$

If we keep the condition (26) together with a similar condition for this new system, i. e.

$$
\bar{b}_{2}=\frac{\bar{b}_{3}+\bar{b}_{5}}{2}+2 \bar{b}_{0}, \quad \bar{a}_{1}=-\frac{\bar{b}_{3}+\bar{b}_{5}}{2}+2 \bar{b}_{0},
$$

then we have

$$
a_{4}+2 b_{3}=0 .
$$

This implies $\bar{a}_{4}+2 \bar{b}_{3}=0$. Comparing with (34), we find $\gamma=\bar{\gamma}=0$, hence it is impossible to construct $(2,2)$-distributions of limit cycles, and this fact was proved by P. Zhang in general (see [8]).

If we make suitable additional perturbation, then it is possible to construct $(2,1)$-distributions of limit cycles. In fact we use formula (25) for the system (37), then we find the first order averaging for another annulus is

$$
\bar{f}^{10}(R)=\zeta_{1} R^{3}+\zeta_{2} R
$$

where

$$
\zeta_{1}=\bar{b}_{2}-\frac{\bar{b}_{3}+\bar{b}_{5}}{2}-2 \bar{b}_{0}, \quad \zeta_{2}=\frac{\bar{a}_{1}+\bar{b}_{2}}{2}-2 \bar{b}_{0}
$$


By the equalities (38) we have that

$$
\zeta_{1}=0, \quad \zeta_{2}=-\frac{a_{4}+2 b_{3}}{2} .
$$

To obtain 2 hyperbolic limit cycles surrounding the singularity in upper half plane for sufficiently small $\varepsilon$, we need the condition that the Jacobian (35) is different from zero, which implies $\zeta_{2} \neq 0$. Now we change $b_{2}$ to $b_{2}+\delta$, where $\delta$ sufficiently small: $0<|\delta| \ll|\varepsilon|$, such that the hyperbolic limit cycles still exist, and $0<\left|\zeta_{1}\right| \ll\left|\zeta_{2}\right|, \zeta_{1} \zeta_{2}<0$ in formula (41), and this gives $(2,1)$-configuration of limit cycles.

The other configuration of limit cycles stated in Theorem 2 can be constructed similarly.

Acknowledgements C. Li wants to thank to the CRM and to the Department of Mathematics of the Universitat Autònoma de Barcelona for their support and hospitality. This paper is also supported by the CRM Research Program: On Hilbert's 16th Problem.

\section{REFERENCES}

[1] A. Buič And J. LliBre, Averaging methods for finding periodic orbits via Brouwer degree, Bull. Sci. Math. 128 (2004), 7-22.

[2] C. Chicone And M. JacoBs, Bifurcation of limit cycles from quadratic isochrones, J. Differential Equations 91 (1991), 268-326.

[3] I. D. ILIEv, Perturbations of quadratic centers, Bull. Sci. Math. 122 (1998), 107-161.

[4] J. Luibre, Averaging theory and limit cycles for quadratic systems, Radovi Math. 11 (2002), 1-14.

[5] W.S. Loud, Behavior of the period of solutions of certain plane autonomous systems near centers, Contributions to Differential Equations 3 (1964), 21-36.

[6] J. A. Sanders and F. Verhulst, Averaging Methods in Nonlinear Dynamical Systems, Appl. Math. Sci. 59, Springer, 1985.

[7] F. Verhulst, Nonlinear Differential Equations and Dynamical Systems, Universitext, Springer, 1991.

[8] P. Zhang, On the distribution and number of limit cycles for quadratic systems with two foci, Qual. Theory Dyn. Syst. 3 (2002), 437-463

[9] H. ŻOŁA̧DEK, Quadratic systems with center and their perturbations, J. Differential Equations 109 (1994), 223-273.

1 School of Mathematical Sciences, Peking university, Beijing 100871, China E-mail address: licz@math.pku.edu.cn

2 Departament de Matemàtiques, Universitat Autònoma de Barcelona, 08193 Bellaterra, Barcelona, Catalonia, Spain

E-mail address: jllibre@mat.uab.es 